\title{
Método de Estimação de Parâmetros para Modelagem no Domínio Wavelet do Tráfego de Redes de Computadores Usando o Algoritmo de Levenberg-Marquardt
}

\author{
Maykon Renan P. da Silva ${ }^{1}$, Flávio Geraldo C. Rocha ${ }^{2}$ \\ ${ }^{1,2}$ Escola de Engenharia Elétrica, Mecânica e de Computação \\ Universidade Federal de Goiás (UFG) \\ maykonrenan@discente.ufg.br, flaviogcreufg.br
}

\begin{abstract}
In this work proposes a multifractal model based on a multiplicative cascade in the wavelet domain, to synthesize network traffic samples. For this purpose, in the proposed model, a parametric modeling based on an exponential function is used for the variance of the multipliers along the stages of the cascade. The exponential function parameters are obtained through the solution of a non-linear system, for this purpose, the Levenberg-Marquardt method is used. The main contribution of the proposed algorithm is to use a fixed and reduced number of parameters to generate network traffic samples that have characteristics such as self-similarity and wide Multifractal Spectrum Width (MSW) similar to the real network traffic traces.
\end{abstract}

\begin{abstract}
Resumo. Este trabalho propõe um modelo multifractal baseado em uma cascata multiplicativa no domínio wavelet, para sintetizar amostras de tráfego de redes. No modelo proposto, utiliza-se uma modelagem paramétrica baseada em uma função exponencial para a variância dos multiplicadores ao longo dos estágios da cascata. Os parâmetros da função exponencial são obtidos através da solução de um sistema não linear, para este fim, utiliza-se o método de Levenberg-Marquardt. A principal contribuição do algoritmo proposto é utilizar um número fixo e reduzido de parâmetros para gerar amostras de tráfego de redes que apresentem características como autossimilaridade e ampla Largura de Espectro Multifractal (MSW) similares as séries reais de tráfego de redes.
\end{abstract}

\section{Introdução}

Os fluxos de redes de computadores podem englobar diferentes protocolos e conteúdos provenientes de diversas tecnologias de acesso ao meio de comunicação. A heterogeneidade dessas redes proporciona um comportamento de tráfego variável, com presença de rajadas difíceis de serem previstas. A melhoria no desempenho das redes de comunicações tem sido alvo de pesquisas constantes, e muitos são os trabalhos propondo modelos para descrição do tráfego de redes [Riedi et al. 1999, Krishna et al. 2003, Millán and Lefranc 2015, Tuberquia-David et al. 2016, Grandemange et al. 2017].

Dentre os modelos existentes para a caracterização do tráfego de redes de comunicações, modelos autossimilares e multifractais têm recebido grande atenção devido aos seus desempenhos em termos de análise e modelagem relacionados ao tráfego real de redes. Os modelos multifractais baseados em cascatas multiplicativas podem ser mais precisos e abrangentes quando aplicados ao controle do tráfego [Feldmann et al. 
1998], [Vieira and Lee 2010], já que em situações práticas o grau de autossimilaridade do tráfego é variante com o tempo, o que não ocorre em modelos Gaussianos e monofractais, reduzindo o horizonte de aplicação destes modelos. Estas propriedades de séries temporais, que vão além daquelas descritas pelos fractais, influenciaram o surgimento de propostas baseadas em multifractais. Desta forma, com uma caracterização do tráfego mais detalhada utilizando análise multifractal, espera-se obter melhores estimativas para a modelagem do tráfego de redes de comunicações.

No entanto, os modelos multifractais podem ser complexos e suas aplicações práticas podem ser limitadas pelo número de seus parâmetros, que geralmente é alto e dependente do número de amostras de tráfego de redes que se deseja modelar.

Neste trabalho propõe-se um modelo multifractal baseado em um número reduzido (quando comparado a outros modelos presentes na literatura) e fixo (não dependente do número de amostras de tráfego a ser modelado) de parâmetros.

O restante do artigo está organizado da seguinte forma: a Seção 2 apresenta uma breve descrição da análise multifractal aplicada ao tráfego de redes; a Seção 3 apresenta alguns dos modelos multifractais presentes na literatura; na Seção 4 é proposto o modelo multifractal proposto baseado na caracterização da variância dos multiplicadores de uma cascata multiplicativa no domínio wavelet; na Seção 5 é avaliada a eficiência do modelo proposto em comparação a outros modelos presentes na literatura: $\beta$ MWM (Multifractal Wavelet Model) [Riedi et al. 1999], VVGM (Variable Variance Gaussian Model) [Krishna et al. 2003] e MFHSW (MultiFractal Hurst Spectrum Width) [Tuberquia-David et al. 2016], utilizando dados reais; na Seção 6 são apresentadas as conclusões obtidas.

\section{Análise Multifractal do Tráfego de Redes}

Fractais são objetos geométricos que possuem uma forma irregular que persiste em várias resoluções de observação [Mandelbrot 1990], como mostra a Figura 1. A maioria dos sinais fractais conhecidos possuem características como a autossimilaridade. O conceito de autossimilaridade está associado aos objetos cuja forma não muda em decorrência da mudança de escala, característica esta que pode influir, por exemplo, no desempenho do tráfego de redes [Vieira and Lee 2010].

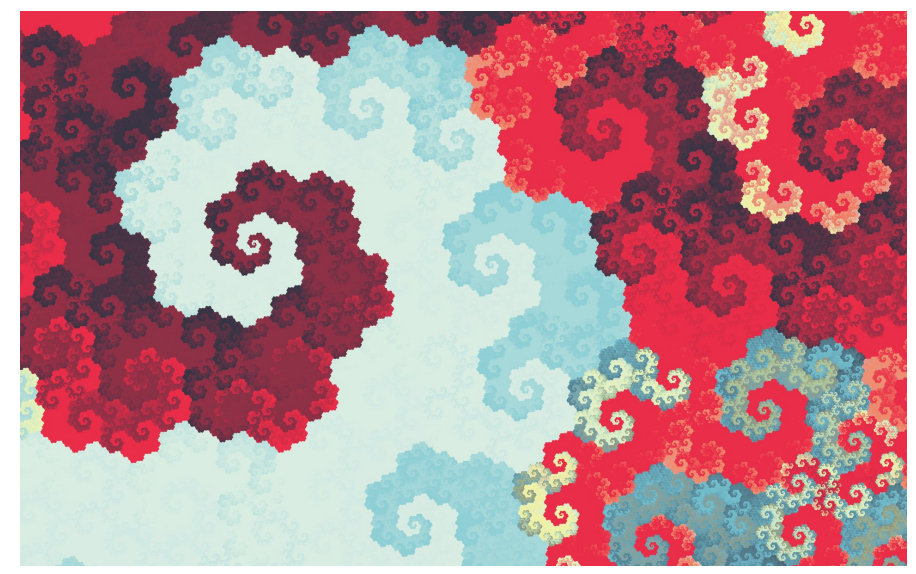

Figura 1. Fractal baseado no conjunto de Mandelbrot [CODERS 2020].

O parâmetro de Hurst $(H)$ mede o grau de autossimilaridade do processo com ca- 
racterística fractal, onde $H$ está contido no intervalo $(0<H<1,0)$. Convém ressaltar que no caso de processos assintoticamente autossimilares restringindo-se o parâmetro de Hurst ao intervalo $(0,5<H<1,0)$, autossimilaridade implica em longa dependência. Assim, diz-se que processos autossimilares com $H>0,5$ apresentam dependência de longa duração; isto significa que a função de autocorrelação (ACF - Autocorrelation Function) do processo apresenta decaimento hiperbólico [Abry and Veitch 1998].

A autossimilaridade introduz dificuldades na otimização do desempenho da rede e na garantia de qualidade de serviço (QoS), podendo causar impactos significativos como aumento da latência e da taxa de perda de pacotes. Um conceito relacionado à autossimilaridade é o de distribuição de cauda pesada (heavy tail) onde a densidade de probabilidade dos processos de tráfego decai lentamente [Park and Willinger 2000]. Quanto mais o tráfego de uma rede apresenta características de uma distribuição de cauda pesada, mais os valores de parâmetros de QoS dessa rede são degradados, [Rocha 2011].

Assim como os fractais, os multifractais são utilizados em muitas áreas como análise de fenômenos naturais como terremotos [Barman et al. 2015], processamento e decomposição de sinais [Mallat 2008] e modelagem de tráfego de redes de computadores [Riedi et al. 1999], [Krishna et al. 2003], [Tuberquia-David et al. 2016]. Na análise multifractal verifica-se o comportamento em escala de momentos estatísticos dos processos para estimar suas regularidades locais [Feldmann et al. 1998], [Riedi et al. 1999]. Através de ferramentas da análise multifractal algumas propriedades encontradas em processos reais podem ser verificadas. O tráfego de redes, por exemplo, ao ser considerado multifractal significa que possui uma estrutura de forte dependência inerente entre as amostras, com incidência de rajadas em várias escalas [Riedi et al. 1999]. Processos multifractais são definidos a seguir, [Vieira et al. 2012].

Definição 2.1. Um processo estocástico $X(t)$ é multifractal se satifaz a equação:

$$
\left.\left.\mathbb{E}(\mid X(t))\right|^{q}\right)=c(q) t^{\tau(q)+1},
$$

onde $t \in T$ e $q \in Q$ são intervalos na reta real, $\tau(q)$ e $c(q)$ são funções no domínio $Q$. Normalmente, assume-se $T$ e $Q$ tenham comprimentos positivos, e que $0 \in T,[0,1] \subseteq Q$.

A Definição 2.1 permite que "multifractalidade" seja descrita em termos de momentos, onde $\tau(q)$ é a função de escala e $c(q)$ é o fator de momento de um processo multifractal. Caso $\tau(q)$ seja linear em $q$ o processo é dito ser monofractal, caso contrário, é multifractal [Riedi et al. 1999], [Park and Willinger 2000], [Vieira et al. 2012].

\section{Trabalhos Relacionados}

O modelo multifractal MWM é baseado em uma cascata multiplicativa no domínio wavelet [Riedi et al. 1999], tendo sido recentemente utilizado para a modelagem do tráfego de redes [Tuberquia-David et al. 2016]. No MWM, a variável $U_{j, k}$ representa os coeficientes de escala; $W_{j, k}$ representa os coeficientes wavelets e $A_{j, k}$ representa os multiplicadores da cascata multiplicativa; o índice $j$ representa o estágio da cascata (também chamado de escala) e $k$ é o instante de tempo dentro da escala $j$.

Para se gerar um processo segundo o modelo MWM é preciso aplicar a transformada wavelet discreta (DWT - Discrete Wavelet Transform) ao tráfego de redes, calcular os momentos de segunda ordem dos coeficientes wavelet $\left(W_{j, k}\right)$ em cada escala da cascata, a média e a variância dos coeficientes de escala $\left(U_{j, k}\right)$ na escala de menor resolução e 
calcular $\rho_{j}$, variável usada para capturar o decaimento de energia dos coeficientes wavelet $\left(W_{j, k}\right)$ em função da escala $j$. A Figura 2 ilustra como é construída a cascata multiplicativa no domínio wavelet a partir dos parâmetros descritos anteriormente.

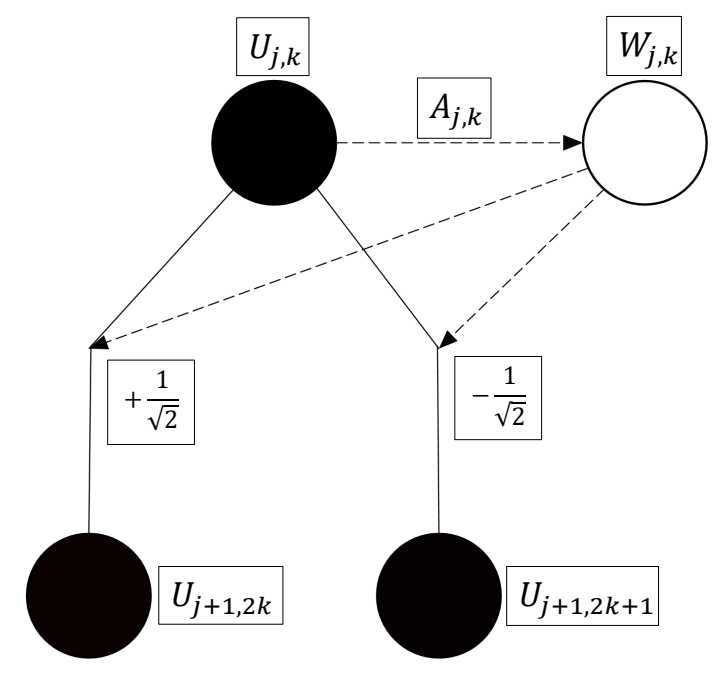

Figura 2. Cascata multiplicativa formada pelos coeficientes de escala $U_{j, k}$, coeficientes wavelet $W_{j, k}$ e pelos multiplicadores $A_{j, k}$.

Quando os multiplicadores $A_{j, k}$ podem ser descritos por meio de uma distribuição $\beta(\cdot, \cdot)$, o modelo MWM é chamado de $\beta$ MWM.

No $\beta$ MWM o número de parâmetros para gerar uma série sintética de tráfego de redes depende do tamanho da série real de tráfego, ou seja quanto maior o número de amostras da série real de tráfego, maior o número de escalas $n$ necessárias para se construir a cascata multiplicativa e consequentemente maior o comprimento do vetor $\rho_{j}$ $(j=0,1,2, \ldots, n-1)$. Assim, o número de parâmetros necessários para gerar um processo de tráfego no $\beta$ MWM é de $(n+2)$, onde $n$ é o número de termos do vetor $\rho_{j}$ e os parâmetros $\mu_{c}$ e $\sigma_{c}$ correspondem a média e ao desvio padrão do coeficiente de escala $U_{0,0}$, respectivamente, gerado segundo uma distribuição Gaussiana.

O modelo VVGM [Krishna et al. 2003] baseia-se em uma cascata multiplicativa onde os valores correspondentes aos multiplicadores da cascata são amostras de uma distribuição Gaussiana a cada estágio da cascata, ou seja, nesse modelo assume-se que a distribuição dos multiplicadores é Gaussiana com média $(\mu)$ igual a 0.5 e variância $\left(\sigma_{j}\right)$ que varia a cada escala da cascata, a medida que ela é sintetizada a partir do parâmetro $x^{N}$ que representa o valor agregado das $N$ amostras de tráfego.

O VVGM atribui um valor fixo de média para os multiplicadores de todas as escalas o que pode comprometer o processo de modelagem, visto que séries reais de tráfego de redes apresentam multiplicadores com média variável a cada escala. Assim como o $\beta$ MWM, o VVGM requer $(n+2)$, parâmetros para a síntese de amostras de tráfego de redes . Maiores detalhes sobre o VVGM podem ser obtidos em [Krishna et al. 2003].

O modelo MFHSW [Tuberquia-David et al. 2016] permite ao projetista de redes a configuração a priori dos parâmetros de $\operatorname{Hurst}(H)$ e de largura do espectro multifractal (MSW - Multifractal Spectrum Width). Assim como o $\beta$ MWM, o MFHSW baseia-se 
em uma cascata multiplicativa no domínio wavelet e o número de parâmetros necessários para gerar uma série sintética de tráfego está diretamente ligado ao número de amostras da série real de tráfego, além de ser necessário estimar os parâmetros $\mu_{c}, \sigma_{c}$ e $\rho_{j}$, o MFHSW precisa que o projetista ajuste os parâmetros $H$ e $M S W$, o que torna o MFHSW um algoritmo que precisa de $(n+5)$ parâmetros para gerar um processo de tráfego sintético. Não obstante, caso a série sintética obtida não atenda aos parâmetros $H$ e $M S W$ predeterminados, essa série é descartada e o algoritmo é reiniciado, o que melhora a precisão do modelo, mas aumenta consideravelmente o tempo de execução do algoritmo.

\section{Modelo Multifractal Proposto}

Nesta seção, apresenta-se a Proposição 1, e sua respectiva demonstração, que fundamenta o modelo proposto. A Proposição 1 baseia-se em uma estrutura de cascata multiplicativa no domínio wavelet onde a variância dos multiplicadores ao longo das escalas da cascata é modelada por meio de uma função exponencial. Para tanto, na nova abordagem proposta, os valores de $\rho_{j}$, que estão relacionados com o decaimento de energia dos coeficientes wavelets do modelo $\beta \mathrm{MWM}$, são calculados de forma paramétrica ao se modelar os momentos de segunda ordem dos multiplicadores da cascata $\left(\mathbb{E}\left[A_{(j)}^{2}\right]\right)$ a partir do parâmetro de Hurst $(H)$. Em seguida apresenta-se dois algoritmos que constituem o modelo proposto: o Algoritmo 1 (Estimação de Parâmetros) responsável pela modelagem dos parâmetros do modelo proposto com base na estrutura da cascata multiplicativa no domínio wavelet, como mostra a Figura 2; o Algoritmo 2 (Síntese de Tráfego) responsável por gerar amostras sintéticas de tráfego de redes com base nos parâmetros estimados pelo Algoritmo 1.

Proposição 1. Uma série temporal de tráfego real de redes $C(t)$ pode ser descrita por um processo sintético $C^{(n)}[k]$ gerado por meio do modelo proposto que é baseado em três parâmetros: média $\left(\mu_{c}\right)$ e desvio padrão $\left(\sigma_{c}\right)$ do coeficiente de escala $U_{0,0}$ e parâmetro de Hurst $(H)$. A taxa de distribuição $(\alpha)$, parâmetro de forma $(\beta)$ e o parâmetro de localização $(\gamma)$ de uma função exponencial utilizada para modelar a variância dos multiplicadores $A_{(j)}$ são obtidos a partir do valor de $H$. O processo sintético é definido pela seguinte equação:

$$
\begin{aligned}
C^{(n)}[k] & \stackrel{\mathrm{d}}{=} 2^{-n} G\left(\mu_{c}, \sigma_{c}\right)\left[1+\beta\left(\frac{2-4^{H}}{4^{H}-4}, \frac{2-4^{H}}{4^{H}-4}\right)\right] \times \\
\times & \prod_{j=0}^{n-2}\left[1+\beta\left(\frac{e^{\left(\frac{j+1-\beta}{\gamma}\right)^{2}}-\alpha}{2 \alpha}, \frac{e^{\left(\frac{j+1-\beta}{\gamma}\right)^{2}}-\alpha}{2 \alpha}\right)\right],
\end{aligned}
$$

onde $k=0,1, \ldots, 2^{j-1}$ é o deslocamento de tempo para $j=0,1, \ldots, n-1$ e $n$ é a variável relacionada à escala de tempo mais fina (de maior resolução) de uma cascata multiplicativa no domínio wavelet.

Demonstração. O processo para se gerar uma série sintética de tráfego de redes pode ser definido pela seguinte equação [Riedi et al. 1999]:

$$
C^{(n)}[k] \stackrel{\mathrm{d}}{=} 2^{-n} U_{0,0} \prod_{j=0}^{n-1}\left[1+A_{(j)}\right],
$$


onde $U_{0,0} \stackrel{\mathrm{d}}{=} G\left(\mu_{c}, \sigma_{c}\right)$ e $A_{(j)}$ representa $A_{j, k}$, pois os multiplicadores podem ser descritos por uma distribuição $\beta$ simétrica, onde $A_{(j)} \stackrel{\text { d }}{=} \beta\left(\rho_{j}, \rho_{j}\right)$. Os valores de $\beta\left(\rho_{j}, \rho_{j}\right)$ estão contidos no intervalo $[-1,1]$.

A partir do parâmetro de Hurst $(H)$ calcula-se o parâmetro $\rho_{j}$ para $j=n-1$ (última escala), da seguinte forma [Riedi et al. 1999]:

$$
\rho_{n-1}=\frac{2^{2 H-1}-1}{2-2^{2 H-1}}
$$

onde,

$$
\lim _{H \rightarrow 0,5} \rho_{n-1}=0 \quad e \lim _{H \rightarrow 1,0} \rho_{n-1}=\infty,
$$

$\log 0, \forall H \in \mathbb{R}, \exists \epsilon>0 \mid \lim _{\epsilon \rightarrow 0} H \in[0,5+\epsilon ; 1,0-\epsilon]$.

Pode-se calcular a variância de $A_{(j)}$ em função de $\rho_{j}$ pela seguinte relação [Riedi et al. 1999]:

$$
\begin{aligned}
\mathbb{E}\left[A_{(j)}^{2}\right]-\mathbb{E}\left[A_{(j)}\right]^{2} & =\frac{1}{2 \rho_{j}+1}, \\
\mathbb{E}\left[A_{(j)}^{2}\right] & =\frac{1}{2 \rho_{j}+1},
\end{aligned}
$$

onde $\mathbb{E}\left[A_{(j)}\right]=0$. Substituindo (4) em (5) pode-se calcular $\mathbb{E}\left[A_{(j)}^{2}\right]$ para a última escala, pela seguinte equação:

$$
\mathbb{E}\left[A_{(n-1)}^{2}\right]=\frac{1}{2 \frac{2^{2 H-1}-1}{2-2^{2 H-1}}+1}=2^{2-2 H}-1 .
$$

Assumindo que para $j=0$ a cascata multiplicativa na primeira escala é formada por apenas um multiplicador $A_{(0)}$, a variância de apenas um termo é teoricamente zero. $\mathrm{Na}$ abordagem proposta assume-se que a variância é infinitesimal e definida por:

$$
\mathbb{E}\left[A_{(0)}^{2}\right]=\epsilon
$$

Na prática, o valor de $\epsilon$ representa o grau de variabilidade entre os valores agregados $\left(U_{0,0}\right)$ de tempos entre chegadas de amostras de tráfego de redes. Assumindo que a variância dos multiplicadores pode ser modelada por uma função exponencial de três parâmetros $(\alpha . \beta$ e $\gamma)$, obtém-se:

$$
\mathbb{E}\left[A_{(j)}^{2}\right]=\alpha e^{-\left(\frac{j+1-\beta}{\gamma}\right)^{2}}, \text { para } j=0,1, \ldots, n-1 .
$$

Substituindo (6) e (7) em (8), obtém-se:

$$
\left\{\begin{array}{lll}
\alpha e^{-\left(\frac{n-\beta}{\gamma}\right)^{2}} & = & 2^{2-2 H}-1, \\
\alpha e^{-\left(\frac{1-\beta}{\gamma}\right)^{2}}= & \epsilon
\end{array}\right.
$$

Tendo $H$ como parâmetro de entrada, soluciona-se (9) para obter $\alpha, \beta$ e $\gamma$. Entretanto, como (9) não pode ser solucionado diretamente, utiliza-se o método de LevenbergMarquardt (LM) com o objetivo de encontrar os melhores valores para $\alpha, \beta$ e $\gamma$ na parametrização da variância dos multiplicadores. 
O método de Levenberg-Marquardt, ao utilizar o critério dos mínimos quadrados não lineares, oferece um método eficiente para essa parametrização.

Considerando o problema dos mínimos quadrados, dado pela seguinte relação:

$$
\min _{\mathrm{em} \mathbf{p}}\left|\mathbf{J}_{k} \mathbf{p}_{k}+\mathbf{f}_{k}\right|
$$

o método de LM para minimização de resíduos baseia-se no seguinte sistema:

$$
\left(\mathbf{J}_{k}^{T} \mathbf{J}_{k}+\lambda_{k} \mathbf{I}\right) \mathbf{p}_{k}=-\mathbf{J}_{k}^{T} \mathbf{f}_{k},
$$

onde $\mathbf{f}_{k}$ é o vetor de funções não lineares, $\mathbf{J}_{k}$ é a matriz Jacobiana de $\mathbf{f}_{k}, \mathbf{I}_{n \times n}$ é a matriz identidade e $\lambda_{k}$ é um número real não-negativo denominado parâmetro de LevenbergMarquardt [Cunha 2000].

Para ajustar os valores $\left(j, \mathbb{E}\left[A_{(j)}^{2}\right]\right)$ utiliza-se uma função do tipo:

$$
y\left(j ; a_{1}, a_{2}, a_{3}\right)=a_{1} e^{-\left(\frac{j+1-a_{2}}{a_{3}}\right)^{2}} .
$$

onde $a_{1}, a_{2}$ e $a_{3}$ correspondem aos parâmetros $\alpha, \beta$ e $\gamma$, respectivamente.

Nestes cálculos, toma-se a aproximação inicial $\mathbf{a}=(1,1,1)$. Em cada iteração chama-se $\mathbf{a}=\mathbf{a}^{k}$ e calcula-se:

1. Vetor resíduo;

$$
f_{j}(\mathbf{a})=\mathbb{E}\left[A_{(j)}^{2}\right]-a_{1} e^{-\left(\frac{j+1-a_{2}}{a_{3}}\right)^{2}},
$$

2. Matriz jacobiana. As duas colunas dessa matriz são;

$$
\begin{gathered}
J_{j, 1}=\frac{\partial f_{j}}{\partial a_{1}}(\mathbf{a})=e^{-\left(\frac{j+1-a_{2}}{a_{3}}\right)^{2}}, j=0: n-1, \\
J_{j, 2}=\frac{\partial f_{j}}{\partial a_{2}}(\mathbf{a})=\frac{2 a_{1}\left(j+1-a_{2}\right) e^{-\left(\frac{a_{2}-(j+1)}{a_{3}}\right)^{2}}}{a_{3}^{2}}, j=0: n-1, \\
J_{j, 3}=\frac{\partial f_{j}}{\partial a_{3}}(\mathbf{a})=\frac{2 a_{1}\left(j+1-a_{2}\right)^{2} e^{-\left(\frac{j+1-a_{2}}{a_{3}}\right)^{2}}}{a_{3}^{3}}, j=0: n-1 .
\end{gathered}
$$

Com os parâmetros $\alpha, \beta$ e $\gamma$ já estimados por meio de (11) pode-se calcular $\rho_{j}$ para as escalas $j=0,1, \ldots, n-1$. Isolando $\rho_{j}$ em (5), obtém-se:

$$
\rho_{j}=\frac{1}{2}\left(\frac{1}{\mathbb{E}\left[A_{(j)}^{2}\right]}-1\right) .
$$

Substituindo (6) em (17), tem-se $\rho_{j}$ para a última escala:

$$
\rho_{n-1}=\frac{1}{2}\left(\frac{1}{2^{2-2 H}-1}-1\right)=\frac{2-4^{H}}{4^{H}-4} .
$$


Substituindo (8) em (17), tem-se $\rho_{j}$ para as outras escalas da cascata:

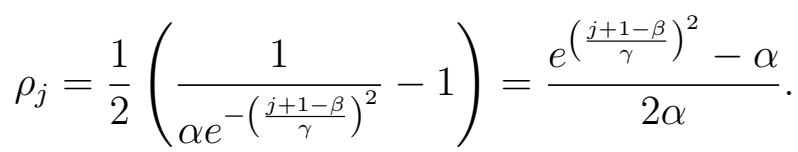

Por fim, substituindo (18) e (19) em (3), obtém-se (2).

No modelo proposto os valores de $\rho_{j}$ para todas as escalas são calculados de forma paramétrica. Por isso, para gerar uma série sintética de tráfego de redes por meio do proposto são necessários apenas três parâmetros $\left(\mu_{c}, \sigma_{c} \mathrm{e} H\right)$, o que faz do modelo proposto um algoritmo cujo o número de parâmetros não depende da quantidade de amostras de tráfego. O modelo proposto é dividido em dois algoritmos apresentados a seguir:

Algoritmo 1 (Estimação de Parâmetros): Estimação dos parâmetros do modelo proposto a partir de uma série real de tráfego de redes $C(t)$.

Entrada: $C(t)$; Saída: $\mu_{c}, \sigma_{c}, H$.

1. Determinar $U_{j, k}$ e $W_{j, k}$ por meio da DWT (Discrete Wavelet Transform), dado que $W_{j, k}=\left\langle C(t), \psi_{j, k}\right\rangle$, onde $\psi_{j, k}$ é a wavelet de Haar;

2. Utilizar a estrutura da cascata multiplicativa obtida no passo 1 para determinar $\mu_{c}$ e $\sigma_{c}$, média e desvio padrão de $U_{0,0}$, respectivamente;

3. Utilizar $W_{j, k}$ para estimar $H$ por meio do diagrama log-escala ( $L D$ - Logscale Diagram) proposto por [Abry and Veitch 1998].

Algoritmo 2 (Síntese de Tráfego): Gerar amostras sintéticas de tráfego de redes a partir dos três parâmetros obtidos na saída do Algoritmo 1.

Entrada: $\mu_{c}, \sigma_{c}, H$; Saída: $C^{(n)}[k]$.

1. Tendo $H$ como parâmetro de entrada, determinar $\alpha, \beta$ e $\gamma$ por meio de (11) (método de Levenberg-Marquardt);

2. Para $j=0$, gerar $U_{0,0}$ de acordo com uma distribuição Gaussiana com parâmetros $\mu_{c}$ e $\sigma_{c}$, i.e., $G\left(\mu_{c}, \sigma_{c}\right)$;

3. Utilizar $\alpha$ e $\gamma$ para calcular $\rho_{j}$ por meio de (18) e (19) para cada escala $j$, e gerar $k$ valores aleatórios de acordo com a distribuição $\beta\left(\rho_{j}, \rho_{j}\right)$ para obter $A_{(j)}$;

4. Utilizar (2) para gerar uma série de amostras sintéticas $\left(C^{(n)}[k]\right)$ de tráfego de redes.

O modelo proposto diferencia-se dos modelos $\beta$ MWM, VVGM e MFHSW, por ser eficiente e não utilizar uma quantidade de parâmetros dependente do número de amostras a serem modeladas. O modelo proposto utiliza um número fixo e reduzido de parâmetros, sendo eles: a média $\left(\mu_{c}\right)$ e desvio padrão $\left(\sigma_{c}\right)$ do tráfego agregado e o parâmetro de Hurst $(H)$. Por meio desses parâmetros, é possível gerar tráfego sintético utilizando o Algoritmo 2.

\section{Resultados}

Foram conduzidas simulações com o objetivo de avaliar o desempenho do modelo proposto em descrever com acurácia as características das séries reais de tráfego de redes. 
São apresentados resultados obtidos utilizando as séries reais BCpAug89 [Danzig et al. 2000] e dec-pkt-2 [Danzig et al. 2000] que, apesar de não serem atuais, são clássicas na literatura. Além disso, são apresentados os resultados utilizando a série de tráfego MultipathTCP [Coninck et al. 2016]. Essas séries de tráfego de redes apresentam amostras de tempo entre chegadas de pacotes dado em segundos. Resultados similares foram obtidos para outras séries reais de tráfego.

A Figura 3 apresenta a variância dos multiplicadores para as séries reais de tráfego BCpAug89, dec-pkt-2 e MultipathTCP e para as séries sintéticas geradas por meio dos modelos $\beta$ MWM, VVGM, MFHSW e proposto.

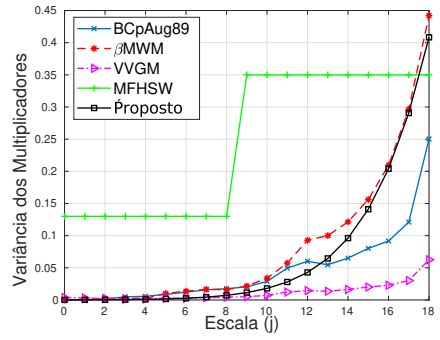

(a)

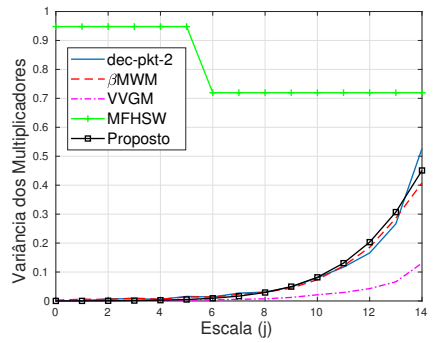

(b)

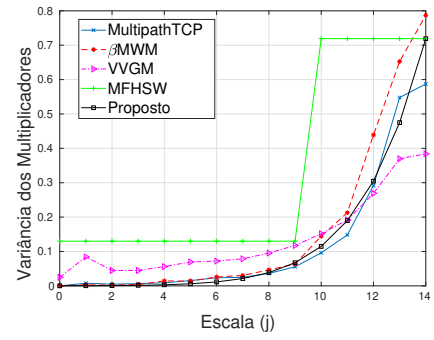

(c)

Figura 3. Variância dos multiplicadores $\left(\mathbb{E}\left[A_{(j)}^{2}\right]\right)$.

A respeito dos resultados para a variância dos multiplicadores, para as séries de tráfego analisadas, o modelo proposto, que utiliza menos parâmetros que os demais modelos, obteve desempenho similar ou superior aos demais, em termos de representação da curva da variância real dos multiplicadores. A Tabela 1 corrobora essa afirmação.

Tabela 1. EQM das curvas da variância dos multiplicadores de cada modelo.

\begin{tabular}{cccc}
\hline Modelos Multifractais & BCpAug89 & dec-pkt-2 & MultipathTCP \\
\hline BMWM & 0,0050 & 0,0021 & 0,0399 \\
VVGM & 0,0155 & 0,0247 & 0,0538 \\
MFHSW & 0,0346 & 0,5437 & 0,0797 \\
Proposto & 0,0029 & 0,0014 & 0,0016 \\
\hline
\end{tabular}

A Tabela 2 apresenta estatísticas e o erro relativo percentual $(E(\%))$ para as séries reais e sintéticas geradas a partir dos modelos considerados. Os resultados da Tabela 2 foram obtidos com uma média de 50 simulações. Analisando os resultados é possível observar que o modelo proposto apresentou o menor $E(\%)$ para o parâmetro de Hurst, além disso apresentou erro apenas ligeiramente maior do que o $\beta \mathrm{MWM}$ para a variância. O VVGM foi o único modelo que apresentou resultados destoantes para a média das amostras das as séries reais de tráfego. O modelo MFHSW obteve o pior desempenho em relação à variância das amostras de tráfego de redes, apresentando um alto $E(\%)$, decorrente de sua ineficiência em estimar a variância dos multiplicadores para diferentes escalas, como mostrou a Figura 3 para diferentes séries reais de tráfego.

Para processos multifractais, o espectro geralmente apresenta uma forma parabólica côncava, cuja a largura $(M S W)$ é proporcional a variabilidade dos expoentes 
Tabela 2. Estatísticas para média, variância e $H$

\begin{tabular}{cccc}
\hline BCpAug89 & Média $\left(10^{-3}\right)$ & Variância $\left(10^{-5}\right)$ & $\boldsymbol{H}$ \\
\hline Real & 3,00 & 2,90 & 0,7156 \\
$\beta$ MWM E(\%) & $3,00(0,0)$ & $2,90(0,0)$ & $0,7275(1,7)$ \\
VVGM E(\%) & $3,30(10,0)$ & $1,48(48,9)$ & $0,7981(11,5)$ \\
MFHSW E(\%) & $3,00(0,0)$ & $58,22(1907,5)$ & $0,7097(0,8)$ \\
Proposto E(\%) & $3,00(0,0)$ & $3,12(7,5)$ & $0,7172(0,2)$ \\
\hline dec-pkt-2 & Média $\left(10^{-3}\right)$ & Variância $\left(10^{-6}\right)$ & $\boldsymbol{H}$ \\
\hline Real & 2,00 & 8,43 & 0,7195 \\
$\beta$ MWM E(\%) & $2,00(0,0)$ & $8,44(0,1)$ & $0,6963(3,2)$ \\
VVGM E(\%) & $2,10(5,0)$ & $9,40(11,5)$ & $0,7432(3,3)$ \\
MFHSW E(\%) & $2,00(0,0)$ & $55,18(554,5)$ & $0,7271(1,05)$ \\
Proposto E $(\%)$ & $2,00(0,0)$ & $8,91(5,7)$ & $0,7124(0,9)$ \\
\hline MultipathTCP & Média $\left(10^{-2}\right)$ & Variância $\left(10^{-4}\right)$ & $\boldsymbol{H}$ \\
\hline Real & 1,11 & 7,78 & 0,6092 \\
$\beta$ MWM E(\%) & $1,11(0,0)$ & $7,82(0,5)$ & $0,6218(2,1)$ \\
VVGM E(\%) & $1,08(2,7)$ & $4,99(35,8)$ & $0,7084(16,3)$ \\
MFHSW E(\%) & $1,11(0,0)$ & $54,00(594,1)$ & $0,6209(1,9)$ \\
Proposto E $(\%)$ & $1,11(0,0)$ & $7,79(0,1)$ & $0,6102(0,8)$ \\
\hline
\end{tabular}

de Hölder [Riedi et al. 1999], [Tuberquia-David et al. 2016]. A Figura 4 apresenta os espectros multifractais para as séries reais de tráfego de redes BCpAug89, dec-pkt-2 e MultipathTCP. O modelo proposto apresentou um espectro largo e com uma concavidade similar ao apresentado pelas séries reais. Essa afirmação é corroborada pela Tabela 3, onde o modelo proposto apresentou o menor $\mathrm{E}(\%)$ para a $M S W$ para as três séries utilizadas. Embora o MFHSW tenha apresentado uma $M S W$ próxima à largura das séries reais, observa-se por meio da Figura 4 que a curva do espectro do modelo MFHSW utilizando a série dec-pkt-2 está deslocada no eixo dos expoentes de Hölder em relação às curvas obtidas para a série real e para o modelo proposto.

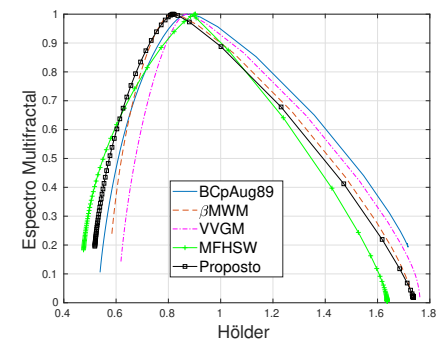

(a)

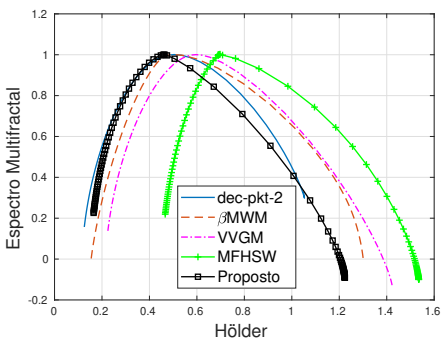

(b)

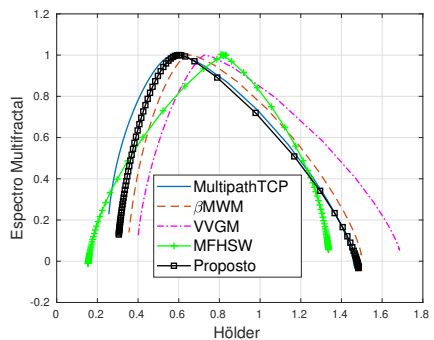

(c)

Figura 4. Espectro multifractal.

A Tabela 4 apresenta os parâmetros necessários para gerar amostras sintéticas de tráfego de redes de acordo com os modelos proposto, $\beta$ MWM, VVGM e MFHSW. Os modelos $\beta$ MWM, VVGM e MFHSW apresentam um vetor de parâmetros, onde o tamanho do mesmo depende do número de escalas $j$ (que está diretamente relacionada com 
Tabela 3. Largura do Espectro Multifractal (MSW).

\begin{tabular}{cccc}
\hline Modelos Multifractais & BCpAug89 & dec-pkt-2 & MultipathTCP \\
\hline Real & 1,1780 & 0,9278 & 1,2057 \\
BMWM E(\%) & $1,1486(2,5)$ & $1,1464(23,6)$ & $1,1420(5,3)$ \\
VVGM E(\%) & $1,1434(2,9)$ & $1,1980(29,1)$ & $1,2845(6,5)$ \\
MFHSW E $(\%)$ & $1,1665(1,0)$ & $1,0685(15,2)$ & $1,1915(1,2)$ \\
Proposto E(\%) & $1,1852(0,6)$ & $1,0588(14,1)$ & $1,1953(0,8)$ \\
\hline
\end{tabular}

a quantidade de amostras de tráfego de rede a serem modeladas), ou seja, quanto maior o número de escalas maior o vetor e consequentemente maior o número de parâmetros. O modelo proposto diferente dos demais modelos comparados apresenta um número fixo e reduzido de parâmetros, que independe do numero de escalas, ou seja, o número de parâmetros independe da quantidade de amostras do tráfego a ser modelado.

Tabela 4. Parâmetros de entrada para a síntese de tráfego de redes de cada Modelo Multifractal.

\begin{tabular}{cc}
\hline Modelos & Parâmetros \\
$\beta$ MWM & $\mu_{c}, \sigma_{c}, \rho_{j}$ \\
VVGM & $x^{N}, \mu \mathrm{e} \sigma_{j}$ \\
MFHSW & $\mu_{c}, \sigma_{c}, \rho_{j}, H, M S W$ \\
Proposto & $\mu_{c}, \sigma_{c}, H$ \\
\hline
\end{tabular}

\section{Conclusões}

Neste trabalho, foi proposto um modelo para caracterização do tráfego de redes com características multifractais. O modelo utiliza uma função exponencial para obter de forma paramétrica a variância dos multiplicadores ao longo das escalas de uma cascata multiplicativa no domínio wavelet. Os parâmetros da função exponencial são obtidos através da solução de um sistema não linear, para este fim, utiliza-se o método de Levenberg-Marquardt. O modelo proposto foi dividido em dois algoritmos, o Algoritmo 1 (Estimação de Parâmetros) é responsável pelo obtenção dos parâmetros do modelo e o Algoritmo 2 (Síntese de Tráfego) é responsável por gerar amostras de tráfego sintético de rede a partir dos parâmetros obtidos no Algoritmo 1.

Os resultados obtidos por meio de simulações utilizando séries reais de tráfego de redes mostraram que o modelo proposto é eficiente em descrever estatísticas de primeira e segunda ordem (média e variância, respectivamente), parâmetro de Hurst e também a largura do espectro multifractal $(M S W)$. O modelo proposto foi comparado aos modelos $\beta$ MWM, VVGM e MFHSW. Ao contrário dos demais modelos comparados, independente do número de amostras de tráfego que se deseja modelar, o modelo proposto utiliza um número fixo e reduzido de parâmetros $\left(\mu_{c}, \sigma_{c} \mathrm{e} H\right)$. Essas características tornam o modelo eficiente, mais simples de ser compreendido e mais fácil de ter seus parâmetros armazenados do que os demais modelos comparados.

\section{Referências}

Abry, P. and Veitch, D. (1998). A wavelet analysis of long-range dependent traffic. IEEE Trans. on Information Theory, 44:2-15. 
Barman, C., Chaudhuri, H., Deb, A., and Sinha, B. (2015). The essence of multifractal detrended fluctuation technique to explore the dynamics of soil radon precursor for earthquakes. Natural Hazards, 78(2):855-877.

CODERS, A. (2020). Fractals. https://wall.alphacoders.com. Último acesso em 15-042020.

Coninck, Q. D., Baerts, M., Hesmans, B., and Bonaventurea, O. (2016). Crawdad dataset. https://crawdad.org/uclouvain/mptcp_smartphone/20160304. Último acesso em 12-042020.

Cunha, M. C. C. (2000). Métodos Numéricos. Editora Unicamp, São Paulo, BRA.

Danzig, P., Mogul, J., Paxson, V., and Schwartz, M. (2000). The internet traffic archive. http://ita.ee.lbl.gov/. Último acesso em 12-04-2020.

Feldmann, A., Gilbert, A. C., and Willinger, W. (1998). Data networks as cascades: Investigating the multifractal nature of internet wan traffic. ACM SIGCOMM Computer Communication Review, 28(4):42-55.

Grandemange, Q., Bhujwalla, Y., Gilson, M., Ferveur, O., and Gnaedinger, E. (2017). An as-level approach to network traffic analysis and modelling. IEEE International Conference on Communications (ICC), pages 1-6.

Krishna, M. P., Gadre, V., and Desai, U. B. (2003). Multifractal based network traffic modeling. Kluwer Academic Publishers.

Mallat, S. (2008). A wavelet tour of signal processing. Academic Press, $3^{\text {a }}$ edição edition.

Mandelbrot, B. B. (1990). Limit log normal multifractal measures. In Frontiers of Physics: Landau Memorial Conference, (163):309-340.

Millán, G. and Lefranc, G. (2015). Simple technique of multifractal traffic modeling. In 2015 CHILEAN Conference on Electrical, Electronics Engineering, Information and Communication Technologies (CHILECON), pages 329-332.

Park, K. and Willinger, W. (2000). Self-Similar Network Traffic and Performance Evaluation. John Wiley Sons, Inc. New York, NY, USA, 1st edition.

Riedi, R., Crouse, M., Ribeiro, V., and Baraniuk, R. (1999). A multifractal wavelet model with application to network traffic. IEEE Transaction on Information Theory, 45(3):992-1018.

Rocha, F. G. C. (2011). Modelagem multifractal aplicada à estimação de probabilidade de perda de dados e ao controle de fluxos de tráfego em redes. UFG, Goiânia, GO.

Tuberquia-David, M., Vela-Vargas, F., Lópes-Chávez, H., and Hernández, C. (2016). A multifractal wavelet model for the generation of long-range dependency traffic traces with adjustable parameters. Expert Systems With Applications, 62(2016):373-384.

Vieira, F. H. T. and Lee, L. L. (2010). An admission control approach for multifractal network traffic flows using effective envelopes. International Journal of Electronics and Communications, 64(7):629-639.

Vieira, F. H. T., Rocha, F. G. C., and dos Santos Junior, J. A. (2012). Loss probability estimation and control for ofdm/tdma wireless systems considering multifractal traffic characteristics. Computer Communications, 35(2):263-271. 